\title{
A Geometric Aspect of the Two-Parameter Planar Lorentzian Motions
}

\author{
Gülsüim Yeliz Şentürk (D) and Salim Yüce \\ Department of Mathematics, Faculty of Arts and Sciences, Yildiz Technical University, Istanbul 34220, Turkey
}

Correspondence should be addressed to Gülsüm Yeliz Şentürk; ysacli@yildiz.edu.tr

Received 6 April 2018; Accepted 24 June 2018; Published 19 September 2018

Academic Editor: Jaime Gallardo-Alvarado

Copyright (C) 2018 Gülsüm Yeliz Şentürk and Salim Yüce. This is an open access article distributed under the Creative Commons Attribution License, which permits unrestricted use, distribution, and reproduction in any medium, provided the original work is properly cited.

\begin{abstract}
We examined the moving coordinate systems, the polar axes, the density invariance of the polar axis transformation, and the curve plotter points and the support function of the two-parameter planar Lorentzian motion. Furthermore, we were concerned with the determination of the motion using the polar axes and analyzed the motion when the density of the polar axes is zero.
\end{abstract}

\section{Introduction}

As a branch of physics and a subdivision of classical mechanics, kinematics identifies the possible motion of points, objects (bodies), and systems of objects (bodies) geometrically without consideration of the effects (causes) of the motions. It deals with any motion of any object. For mathematics, kinematics is a bridge connecting geometry, physics, and mechanism, we can say that it means geometry of motion. Moreover, kinematics is important to astrophysics, mechanical engineering, physics, biomechanics and robotics. The geometry of such a one- or two-parameter motion of points, bodies, and systems of bodies has a number of applications in physics, geometric design, and design and trajectory of robotics.

Mathematicians, physicists, and mechanists have investigated a rigid body motion in different ways. In general, if a rigid body moves, both its orientation and position vary with time. In the kinematic meaning, these changes are called rotation and translation, respectively. A rigid body, programmed to move in a plane with a one/two independent degree/degrees of freedom is defined as a one/two-parameter planar motion.

In kinematics, W. Blaschke and H. R. Müller defined the one-parameter planar motions and obtained the relation between absolute, relative, and sliding velocities and accelerations in the Euclidean plane in 1956 [1]. Oneparameter motions on Lorentzian plane and Galilean plane are described by A. A. Ergin and M. Akar and S. Yüce, respectively $[2,3]$. They also gave the relations between the velocities and accelerations. Furthermore, A. A. Ergin investigated Lorentzian moving planes and pole points [4]. One-parameter planar motions are given in affine Cayley Klein planes (CK-planes) by generalizing of the motions in Euclidean, Galilean, and Lorentzian planes in [5]. In [6], the authors expressed the higher-order velocities, accelerations, and poles under the one-parameter planar hyperbolic motions and their inverse motions. The higher-order accelerations and poles are also presented by considering the rotation angle as a parameter of the motion and its inverse motion.

Two-parameter planar motions are investigated with different but equivalent definitions $[1,7,8]$. The two-parameter planar Euclidean motions were introduced by W. Blaschke and H. R. Müller. The polar axes of two-parameter motion, the curve plotter points, the density invariance of the polar axes transformation, the support function of two-parameter motion, the normed coordinate system, and the main oneparameter motion obtained from the two-parameter motion have been studied in the Euclidean plane [1]. Local pictures for a general two-parameter planar motions are investigated by C. G. Gibson, W. Hawes, and C. A. Hobbs in [9] and singularities of a general planar motions with two degrees of freedom are studied by C. G. Gibson, D. Marsh, and Y. Xiang in [10]. 
A general planar Euclidean motion is defined by the equations

$$
\begin{aligned}
& x^{\prime}=x \cos \theta-y \sin \theta-u_{1} \cos \theta+u_{2} \sin \theta, \\
& y^{\prime}=x \sin \theta+y \cos \theta-u_{1} \sin \theta-u_{2} \cos \theta .
\end{aligned}
$$

If the functions $\theta, u_{1}$, and $u_{2}$ are given by continuously differentiable functions of the parameters $t_{1}$ and $t_{2}$, then the motion is called a two-parameter motion. Here, if the parameters $t_{1}$ and $t_{2}$ are functions of $t$, then a one-parameter planar motion is obtained [1, 7]. By accepting a certain asymmetry, we can take $\theta$ as the parameter. In this case, we obtain a special two-parameter planar Euclidean motion [8]. After their contributions, numerous studies conducted hitherto on two-parameter motions in Euclidean and nonEuclidean planes have been examined [11-17]. In these papers, one-parameter planar motions obtained from twoparameter planar motions, the geometric locus of Hodograph of any points, and acceleration poles of the motions are examined in Euclidean, complex, hyperbolic, Lorentzian, and Galilean planes. It is proved that the pole points at any position lie on a line in the fixed and the moving planes. The instantaneous kinematics of a special two-parameter motion was investigated in [18]. Two-parametric motions are given in the Lobatchevski plane in [19]. Besides, the two-parameter motion is used for biomechanical modeling of left ventricle (LV) using cardiac tagged magnetic resonance imaging data in biomedical area and for modeling of effective elastic tensor for cortical bone in biomechanics area [20,21].

The purpose of this paper is to combine the field of twoparameter planar motion with Lorentzian geometry. We aim to develop the theory of the two-parameter planar Lorentzian motion by considering geometric aspects. In the second section, we review some of the standard facts on one- and two-parameter planar motions. In Section 3, which is actually the original part of our study, we intend to motivate our investigation of two-parameter planar Lorentzian motions in the way that W. Blaschke and H. R. Müller examine two-parameter motions in the Euclidean plane. Lorentzian plane geometry has similarities and fundamental differences from Euclidean plane geometry in the large. First of all, we define two-parameter planar Lorentzian motion by the help of hyperbolic statements. And then we analyze the moving coordinate systems, the polar axes, the density invariance of the polar axis transformation, zero-density of polar axes, the curve plotter points, and the support function of the motion. All in all, we are interested in the determination of the motion using the polar axes.

\section{Preliminaries}

Let us firstly examine the basic concepts related to Lorentzian plane.

The Lorentzian plane $L$ is the vector space $\mathbb{R}^{2}$ provided with Lorentzian inner product $\langle,\rangle_{L}$ given by

$$
\langle X, Y\rangle_{L}=x_{1} x_{2}-y_{1} y_{2}
$$

where $X=\left(x_{1}, y_{1}\right)$ and $Y=\left(x_{2}, y_{2}\right) \in \mathbb{R}^{2}$. The Lorentzian norm of $X$ is defined $\|X\|_{L}=\sqrt{\left|\langle X, X\rangle_{L}\right|}$. Since $\langle,\rangle_{L}$ is indefinite metric, a vector in $X \in L$ can have one of three casual characters: it can be space-like if $\langle X, X\rangle_{L}>0$ or $X=0$; time-like if $\langle X, X\rangle_{L}<0$; null (light-like) if $\langle X, X\rangle_{L}=0$ [22].

Two vectors $X, Y$ in the Lorentzian plane are Lorentzian orthogonal if and only if $\langle X, Y\rangle_{L}=0$.

Definition 1. A time-like line (or a space-like line) with respect to the coordinate system $\left\{O ; \mathbf{l}_{1}, \mathbf{l}_{2}\right\}$ in Lorentzian plane will be denoted by $l_{t}\left(\right.$ or $l_{s}$ ) and it is defined by the equation

$$
\begin{aligned}
& x \cosh \Psi-y \sinh \Psi=p \\
& \qquad(\text { or } x \sinh \Psi-y \cosh \Psi=p)
\end{aligned}
$$

with the Hesse-coordinates $(p, \Psi)$, where $p$ is the distance from the line to the origin and the direction angle $\Psi \in \mathbb{R}$ [23].

Definition 2. The measure of a set of points $X=(x, y)$ is defined by the integral, over the set, of the differential forms

$$
d X=d x \wedge d y
$$

which is called the density for points $X[23,24]$.

Definition 3. The measure of a set of non null lines $l(p, \Psi)$ in the Lorentzian plane is defined by the integral, over the set, of the differential form

$$
d l=d p \wedge d \Psi,
$$

which is called the density for non null lines $l$ [23].

Let us talk about one-parameter motions in Lorentzian and hyperbolic planes and examine the two-parameter planar Lorentzian motions. It is necessary to describe the oneparameter planar motions in order to obtain the twoparameter planar motions. The classical reference on kinematics is [1]. In this book, W. Blaschke and H. R. Müller defined one-parameter motions in the Euclidean and complex planes. A. A. Ergin considered the Lorentzian plane instead of the Euclidean plane and defined the one-parameter planar Lorentzian motions [2]. He also gave the relations between absolute, relative, and sliding velocities (and accelerations) in 1991.

2.1. One-Parameter Motions in Lorentzian and Hyperbolic Planes. Let us firstly give one-parameter motions in Lorentzian plane.

Definition 4. Let $L$ and $L^{\prime}$ be moving and fixed Lorentzian planes. Let $\left\{O ; \mathbf{l}_{1}, \mathbf{l}_{2}\right\}$ and $\left\{O^{\prime} ; \mathbf{l}_{1}{ }^{\prime}, \mathbf{l}_{2}{ }^{\prime}\right\}$ be their orthonormal coordinate systems, respectively. Let us take the points $X=$ $(x, y)$ and $X^{\prime}=\left(x^{\prime}, y^{\prime}\right)$ with respect to the moving and fixed coordinate systems, respectively, and $\mathbf{O O}^{\prime}=\mathbf{u}=u_{1} \mathbf{l}_{1}+u_{2} \mathbf{l}_{2}$. A general planar Lorentzian motion is given by the equations

$$
\begin{aligned}
& x^{\prime}=x \cosh \Theta+y \sinh \Theta-u_{1} \cosh \Theta-u_{2} \sinh \Theta, \\
& y^{\prime}=x \sinh \Theta+y \cosh \Theta-u_{1} \sinh \Theta-u_{2} \cosh \Theta .
\end{aligned}
$$


If $\Theta, u_{1}$, and $u_{2}$ are given by continuously differentiable functions of a time parameter $t$, then the motion is called oneparameter planar Lorentzian motion. We will use $H_{I}=L / L^{\prime}$ to denote one-parameter planar motion in the Lorentzian plane [2].

A. A. Ergin also studied three Lorentzian planes and investigated relative, sliding, and absolute velocities [4]. Let $\mathscr{A}$ and $L$ be moving Lorentzian planes and $L^{\prime}$ be fixed Lorentzian plane and $\left\{A ; \mathbf{b}_{1}, \mathbf{b}_{2}\right\},\left\{O ; \mathbf{l}_{1}, \mathbf{l}_{2}\right\}$, and $\left\{O^{\prime} ; \mathbf{l}_{1}{ }^{\prime}, \mathbf{l}_{2}{ }^{\prime}\right\}$ be their coordinate systems, respectively. Assume that $\Phi$ and $\Phi^{\prime}$ are rotation angles of one-parameter planar Lorentzian motions $\mathscr{A} / L$ and $\mathscr{A} / L^{\prime}$. In [4], the pole points of the Lorentzian motions $\mathscr{A} / L$ and $\mathscr{A} / L^{\prime}$ are examined. Let $X=(x, y)$ be a moving point on the plane $\mathscr{A}$. Since the vector equations

$$
\begin{aligned}
\mathbf{A X} & =x \mathbf{b}_{1}+y \mathbf{b}_{2}, \\
\mathbf{A O} & =\mathbf{a}=a_{1} \mathbf{b}_{1}+a_{2} \mathbf{b}_{2}, \\
\mathbf{A} \mathbf{O}^{\prime} & =\mathbf{a}^{\prime}=a_{1^{\prime}} \mathbf{b}_{1}+a_{2^{\prime}} \mathbf{b}_{2},
\end{aligned}
$$

can be written, we have

$$
\begin{gathered}
\mathbf{x}=\mathbf{O X}=\mathbf{O A}+\mathbf{A X}=x \mathbf{b}_{1}+y \mathbf{b}_{2}-\mathbf{a}, \\
\mathbf{x}^{\prime}=\mathbf{O}^{\prime} \mathbf{X}=\mathbf{O}^{\prime} \mathbf{A}+\mathbf{A X}=x \mathbf{b}_{1}+y \mathbf{b}_{2}-\mathbf{a}^{\prime}
\end{gathered}
$$

Assume that $d$ denotes the differential with respect to the Lorentzian plane $L$ and $d^{\prime}$ denotes the differential with respect to the Lorentzian plane $L^{\prime}$. For the sake of shortness let us use

$$
\begin{aligned}
\Delta & =d \Phi \\
\Sigma_{1} & =d a_{1}+a_{2} \Delta, \\
\Sigma_{2} & =d a_{2}+a_{1} \Delta, \\
\Delta^{\prime} & =d \Phi^{\prime}, \\
\Sigma_{1}{ }^{\prime} & =d a_{1}{ }^{\prime}+a_{2}{ }^{\prime} \Delta^{\prime}, \\
\Sigma_{2}{ }^{\prime} & =d a_{2}{ }^{\prime}+a_{1}{ }^{\prime} \Delta^{\prime} .
\end{aligned}
$$

Hence we can give the following definition.

Definition 5. $\Delta, \Delta^{\prime}, \Sigma_{j}$, and $\Sigma_{j}^{\prime}$ are called the Pfaff forms of one-parameter planar Lorentzian motion with respect to the time parameter $t$, where $1 \leq j \leq 2$.

The derivative equations of the motion $\mathscr{A} / L$ are

$$
\begin{aligned}
d \mathbf{b}_{1} & =\Delta \mathbf{b}_{2}, \\
d \mathbf{b}_{2} & =\Delta \mathbf{b}_{1}, \\
d \mathbf{a} & =\Sigma_{1} \mathbf{b}_{1}+\Sigma_{2} \mathbf{b}_{2},
\end{aligned}
$$

and the derivative equations of the motion $\mathscr{A} / L^{\prime}$, by taking $d^{\prime} \mathbf{a}=d^{\prime} \mathbf{a}^{\prime}$, are

$$
\begin{aligned}
& d^{\prime} \mathbf{b}_{1}=\Delta^{\prime} \mathbf{b}_{2}, \\
& d^{\prime} \mathbf{b}_{2}=\Delta^{\prime} \mathbf{b}_{1}, \\
& d^{\prime} \mathbf{a}=\Sigma_{1}^{\prime} \mathbf{b}_{1}+\Sigma_{2}{ }^{\prime} \mathbf{b}_{2} .
\end{aligned}
$$

We write differentiation of the point $X$ in the plane $L$ as

$$
d \mathbf{x}=\left(d x-\Sigma_{1}+y \Delta\right) \mathbf{b}_{1}+\left(d y-\Sigma_{2}+x \Delta\right) \mathbf{b}_{2} .
$$

Definition 6. The velocity of $X$ with respect to $L$ is called the relative velocity and it is denoted by $\mathbf{V}_{r}=d \mathbf{x} / d t$.

Definition 7. The velocity of $X$ with respect to $L^{\prime}$ is called the absolute velocity and it is denoted by $\mathbf{V}_{a}=d \mathbf{x}^{\prime} / d t$.

If $\mathbf{V}_{r}$ is equal to zero, then $X$ is a fixed point on $L$ and if $\mathbf{V}_{a}$ is equal to zero, then $X$ is a fixed point on $L^{\prime}$. Therefore, the conditions that the point $X$ is fixed in planes $L$ and $L^{\prime}$ can be obtained as follows:

$$
\begin{gathered}
d x=\Sigma_{1}-y \Delta, \\
d y=\Sigma_{2}-x \Delta, \\
d x^{\prime}=\Sigma_{1}{ }^{\prime}-y^{\prime} \Delta^{\prime}, \\
d y^{\prime}=\Sigma_{2}{ }^{\prime}-x^{\prime} \Delta^{\prime} .
\end{gathered}
$$

Definition 8. The expression

$$
\begin{aligned}
d_{f} \mathbf{x}= & \left\{\left(\Sigma_{1}-\Sigma_{1}{ }^{\prime}\right)+y\left(\Delta^{\prime}-\Delta\right)\right\} \mathbf{b}_{1} \\
& +\left\{\left(\Sigma_{2}-\Sigma_{2}{ }^{\prime}\right)+x\left(\Delta^{\prime}-\Delta\right)\right\} \mathbf{b}_{2}
\end{aligned}
$$

is called the sliding velocity vector of the motion and the sliding velocity is defined by $\mathbf{V}_{f}=d_{f} \mathbf{x} / d t$.

The pole point is characterized by vanishing the sliding velocity at the time $t$. Hence, the relative velocity equals the absolute velocity. During the motion, the pole points do not move in both planes. For the pole points $P=\left(p_{1}, p_{2}\right) \in L$ we write

$$
\begin{aligned}
& p_{1}=u_{1}+\frac{d u_{2}}{d \Theta}, \\
& p_{2}=u_{2}+\frac{d u_{1}}{d \Theta}
\end{aligned}
$$

or if we take $d_{f} \mathbf{x}$ as zero, the pole point $P\left(p_{1}, p_{2}\right)$ of the motion is obtained as

$$
\begin{aligned}
& p_{1}=\frac{\Sigma_{2}{ }^{\prime}-\Sigma_{2}}{\Delta^{\prime}-\Delta}, \\
& p_{2}=\frac{\Sigma_{1}{ }^{\prime}-\Sigma_{1}}{\Delta^{\prime}-\Delta} .
\end{aligned}
$$


$X^{\prime}=\left(x^{\prime}, y^{\prime}\right)$ is the image of the point $X$ and

$$
\begin{aligned}
& l_{t}^{\prime} \ldots \quad x^{\prime} \cosh \Psi^{\prime}-y^{\prime} \sinh \Psi^{\prime}=p^{\prime} \\
& l_{s}^{\prime} \ldots \quad x^{\prime} \sinh \Psi^{\prime}-y^{\prime} \cosh \Psi^{\prime}=p^{\prime}
\end{aligned}
$$

are the images of time-like line $l_{t}(p, \Psi)$ and space-like line $l_{s}(p, \Psi)$ under the motion, respectively. Here, $l_{t}^{\prime}\left(p^{\prime}, \Psi^{\prime}\right)$ is defined with help of the direction angle $\Theta+\Psi=\Psi^{\prime}$ and the distance $p^{\prime}=p-u_{1} \cosh \Psi+u_{2} \sinh \Psi$ from the origin $O^{\prime}$ and similarly $l_{s}^{\prime}\left(p^{\prime}, \Psi^{\prime}\right)$ is defined with help of the direction angle $\Theta+\Psi=\Psi^{\prime}$ and the distance $p^{\prime}=p-u_{1} \sinh \Psi+u_{2} \cosh \Psi$ from the origin $O^{\prime}$ [25].

Let us give the one-parameter motions in hyperbolic plane. Motion in hyperbolic plane is congruent to the motion in Lorentzian plane. Because of the fact that there is a strict correspondence between Lorentzian plane and hyperbolic plane similar to the complex plane and Euclidean plane, S. Yüce and N. Kuruoğlu defined one-parameter motions in the hyperbolic plane [26].

Hyperbolic numbers can be introduced as an extension of the real numbers. This extension is obtained by including the hyperbolic imaginary $j$, where $j^{2}=1$ but $j \neq \mp 1$. In this case, the hyperbolic numbers set can be written as follows:

$$
\mathbb{H}=\mathbb{R}[j]:=\left\{z=x+j y \mid x, y \in \mathbb{R}, j^{2}=1\right\} .
$$

The hyperbolic numbers have been also called split-complex numbers, perplex numbers, or double numbers [27]. The collection of all hyperbolic numbers is called the hyperbolic plane $\mathbb{H}$.

Definition 9. Let $\mathbb{H}$ and $\mathbb{U}^{\prime}$ be moving and fixed hyperbolic planes. Let $\left\{O ; \mathbf{h}_{1}, \mathbf{h}_{2}\right\}$ and $\left\{O^{\prime} ; \mathbf{h}_{1}{ }^{\prime}, \mathbf{h}_{2}{ }^{\prime}\right\}$ be their orthonormal coordinate systems, respectively. Then, the one-parameter planar hyperbolic motion is defined by the equation

$$
\mathbf{x}^{\prime}=(\mathbf{x}-\mathbf{u}) e^{j \Theta}
$$

and denoted by $\mathbb{H} / \mathbb{H}^{\prime}$, where $\Theta$ is the rotation angle of the motion and the hyperbolic numbers $\mathbf{x}=x+j y, \mathbf{x}^{\prime}=x^{\prime}+j y^{\prime}$ represent the point $X \in \mathbb{W}$ with respect to the moving and the fixed rectangular coordinate systems, respectively. The hyperbolic number $\mathbf{O}^{\prime} \mathbf{O}=\mathbf{u}^{\prime}=-\mathbf{u} e^{j \Theta}$ represents the origin point $O$ of the moving system in the fixed coordinate system.

Moreover, the angle $\Theta$ and $\mathbf{x}^{\prime}, \mathbf{x}$, and $\mathbf{u}^{\prime}$ are continuously differentiable functions of a time parameter $t$. The vector equation

$$
\mathbf{V}_{a}=\frac{d \mathbf{x}^{\prime}}{d t}=\dot{\mathbf{x}}^{\prime}=j \dot{\Theta} \mathbf{x} e^{j \Theta}-(\dot{\mathbf{u}}+j \mathbf{u} \dot{\Theta}) e^{j \Theta}+\dot{\mathbf{x}} e^{j \Theta}
$$

is called the absolute velocity of the motion. To avoid the pure translation, we assume that $d \Theta / d t=\dot{\Theta} \neq 0$. The vector equation

$$
\mathbf{V}_{f}=j \dot{\Theta} \mathbf{x} e^{j \Theta}-(\dot{\mathbf{u}}+j \mathbf{u} \dot{\Theta}) e^{j \Theta}
$$

is called the sliding velocity of the motion. Suppose that $\mathbf{V}_{f}=0$, and then we get the pole points $P\left(p_{1}, p_{2}\right) \in \mathbb{M}$ and $P^{\prime}\left(p_{1}^{\prime}, p_{2}^{\prime}\right) \in \mathbb{H}^{\prime}$ :

$$
\begin{aligned}
& \mathbf{p}=p_{1}+j p_{2}=\mathbf{u}+j \frac{\dot{\mathbf{u}}}{\dot{\Theta}}, \\
& \mathbf{p}^{\prime}=p_{1}^{\prime}+j p_{2}^{\prime}=j e^{j \Theta} \frac{\dot{\mathbf{u}}}{\dot{\Theta}} .
\end{aligned}
$$

2.2. Two-Parameter Planar Lorentzian Motions. Let us examine the two-parameter planar Lorentzian motions.

Definition 10. In the case of continuously differentiable functions $\Theta, u_{1}$, and $u_{2}$ of the parameters $t_{1}$ and $t_{2}$, (6) determines two-parameter planar Lorentzian motion [12].

Here, if $t_{1}$ and $t_{2}$ are functions of the time parameter $t$, then one-parameter planar Lorentzian motion is obtained. Two-parameter planar Lorentzian motion given by (6) can be written in the form

$$
\begin{aligned}
X^{\prime} & =A X+C, \\
X^{\prime} & =\left[\begin{array}{ll}
x^{\prime} & y^{\prime}
\end{array}\right]^{T}, \\
X & =\left[\begin{array}{ll}
x & y
\end{array}\right]^{T}, \\
C & =\left[\begin{array}{ll}
a & b
\end{array}\right]^{T}
\end{aligned}
$$

where $A \in S O(2,1), a=-u_{1} \cosh \Theta-u_{2} \sinh \Theta, b=$ $-u_{1} \sinh \Theta-u_{2} \cosh \Theta$, and $X^{\prime}$ and $X$ are the position vectors of the same point $X=(x, y)$ and $C$ is the translation vector. By taking derivatives of (23), we get

$$
\mathbf{d} X^{\prime}=d A X+A \mathbf{d} X+d C,
$$

where the velocities $\mathbf{V}_{\mathbf{a}}=\mathbf{d} X^{\prime}, \mathbf{V}_{\mathbf{f}}=d A X+d C$, and $\mathbf{V}_{\mathbf{r}}=\mathbf{d} X$ are called the absolute, the sliding, and the relative velocities of the point $X$, respectively. The solution of the equation $\mathbf{V}_{\mathbf{f}}=$ 0 gives us the pole points $P=\left(p_{1}, p_{2}\right)$. M. K. Karacan and Y. Yaylı investigated one-parameter planar Lorentzian motions obtained from two-parameter planar Lorentzian motion [12]. It is proved that the pole points at any position lie on a line in the fixed and the moving planes and the lengths of the velocity vectors of pole axes are the same. Furthermore, the locus of Hodograph of any point and acceleration poles of the motion are examined.

\section{A New Aspect of the Two-Parameter Planar Lorentzian Motion with regard to the Pole Axes}

This section is the original part of our paper. Our purpose is to give firstly the hyperbolic statement of two-parameter planar Lorentzian motion. After that, we study the moving coordinate systems, the polar axes, the density invariance of the polar axis transformation, the motion with zero-density of the polar axes, the curve plotter points, and the support function. 
3.1. Two-Parameter Planar Lorentzian Motion. Here is another way of describing the two-parameter planar Lorentzian motion by the help of the hyperbolic statements.

Definition 11. Let us consider $u_{1}, u_{2}$, and $\Theta$; namely, $\mathbf{u}=$ $\left(u_{1}, u_{2}\right)$ and $\Theta$ are given by continuously differentiable function of the parameters $t_{1}$ and $t_{2}$ in (19). In this way, we obtain what we call the two-parameter planar Lorentzian motion $L / L^{\prime}$. During this motion, the point $X \in L$ generally draws a surface part in the plane $L^{\prime}$. For this reason, the motion can be called the surface drawing motion. We denote the motion briefly by $H_{I I}$.

Here,

(i) if $t_{1}$ and $t_{2}$ are functions of the time parameter $t$ (parameter is $t$ ),

(ii) if $t_{1}$ is a function of the parameter $t_{2}$ (parameter is $t_{2}$ ),

(iii) if $t_{1}$ is a constant (parameter is $t_{2}$ ),

then one-parameter planar Lorentzian motions $H_{I}$ are obtained.

(iv) If $d \mathbf{u} / d \Theta \neq 0$, then we can take the angle $\Theta$ as a parameter of the motion. Hence, we can write $t_{1}=\Theta$ and $t_{2}=t$. In this case, the motion is called special twoparameter planar Lorentzian motion. We denoted it briefly by $S H_{I I}$. If $t$ is a function of the angle $\Theta$, special one-parameter planar Lorentzian motions $S H_{I}$ are obtained.

During the motion $H_{I}$, obtained from the motion $H_{I I}$, the point $X \in L$ generally draws a curve segment in the plane $L^{\prime}$.

Example 12. The two-parameter planar Lorentzian motion $H_{I I}$

$$
\begin{aligned}
x^{\prime}= & x \cosh \left(t_{1}+t_{2}\right)+y \sinh \left(t_{1}+t_{2}\right) \\
& -t_{1} \cosh \left(t_{1}+t_{2}\right)-t_{2} \sinh \left(t_{1}+t_{2}\right) \\
y^{\prime}= & x \sinh \left(t_{1}+t_{2}\right)+y \cosh \left(t_{1}+t_{2}\right) \\
& -t_{1} \sinh \left(t_{1}+t_{2}\right)-t_{2} \cosh \left(t_{1}+t_{2}\right)
\end{aligned}
$$

describes a surface in the plane $L^{\prime}$, Figure 1.

The one-parameter motions obtained from the motion in (25)

$$
\begin{aligned}
x^{\prime}= & x \cosh 2 t_{1}+y \sinh 2 t_{1}-t_{1} \cosh 2 t_{1} \\
& -t_{2} \sinh 2 t_{1} \\
y^{\prime}= & x \sinh 2 t_{1}+y \cosh 2 t_{1}-t_{1} \sinh 2 t_{1} \\
& -t_{2} \cosh 2 t_{1}, \\
x^{\prime}= & x \cosh t_{1}{ }^{2}+y \sinh t_{1}{ }^{2}-t_{1} \cosh t_{1}{ }^{2}-t_{2} \sinh t_{1}{ }^{2} \\
y^{\prime}= & x \sinh t_{1}{ }^{2}+y \cosh t_{1}{ }^{2}-t_{1} \sinh t_{1}{ }^{2}-t_{2} \cosh t_{1}{ }^{2},
\end{aligned}
$$

describe the red curve and the blue curve in the plane $L^{\prime}$, respectively. These curves can be seen in Figure 1 .

3.2. The Moving Coordinate Systems. It is often necessary to describe the motion by a moving frame and plane.
Let $\mathscr{A}$ and $\mathscr{A}^{\prime}$ be moving Lorentzian planes, $L$ and $L^{\prime}$ be fixed Lorentzian planes, and $\left\{A ; \mathbf{b}_{1}, \mathbf{b}_{2}\right\}$ and $\left\{A^{\prime} ; \mathbf{b}_{1}^{\prime}, \mathbf{b}_{2}^{\prime}\right\}$ and $\left\{O ; \mathbf{l}_{1}, \mathbf{l}_{2}\right\}$ and $\left\{O^{\prime} ; \mathbf{l}_{1}{ }^{\prime}, \mathbf{l}_{2}{ }^{\prime}\right\}$ be their coordinate systems, respectively. We will denote the rotation angles of twoparameter planar Lorentzian motions $\mathscr{A} / L$ and $\mathscr{A}^{\prime} / L^{\prime}$ by $\Phi$ and $\Phi^{\prime}$, respectively.

Here are the derivative equations of the motions $\mathscr{A} / L$ and $\mathscr{A}^{\prime} / L^{\prime}$, respectively:

$$
\begin{aligned}
d \mathbf{b}_{1} & =\Delta \mathbf{b}_{2}, \\
d \mathbf{b}_{2} & =\Delta \mathbf{b}_{1}, \\
d \mathbf{a} & =\Sigma_{1} \mathbf{b}_{1}+\Sigma_{2} \mathbf{b}_{2}, \\
d \mathbf{b}_{1}{ }^{\prime} & =\Delta^{\prime} \mathbf{b}_{2}{ }^{\prime}, \\
d \mathbf{b}_{2}{ }^{\prime} & =\Delta^{\prime} \mathbf{b}_{1}{ }^{\prime}, \\
d \mathbf{a}^{\prime} & =\Sigma_{1}{ }^{\prime} \mathbf{b}_{1}{ }^{\prime}+\Sigma_{2}{ }^{\prime} \mathbf{b}_{2}{ }^{\prime} .
\end{aligned}
$$

Definition 13. $\Delta, \Delta^{\prime}, \Sigma_{j}$, and $\Sigma_{j}^{\prime}$ are called the Pfaff forms of the motions $\mathscr{A} / L$ and $\mathscr{A}^{\prime} / L^{\prime}$. They are the differential forms depending on two-parameter $t_{1}$ and $t_{2}$, where $1 \leq j \leq 2$.

The Pfaff forms cannot be assumed arbitrarily for the motion; they must satisfy the integrability conditions which follow from the external derivation of (27) and (28) by

$$
\begin{gathered}
d \Sigma_{1}=\Sigma_{2} \wedge \Delta, \\
d \Sigma_{2}=\Sigma_{1} \wedge \Delta, \\
d \Sigma_{1}{ }^{\prime}=\Sigma_{2}{ }^{\prime} \wedge \Delta^{\prime}, \\
d \Sigma_{2}{ }^{\prime}=\Sigma_{1}{ }^{\prime} \wedge \Delta^{\prime} .
\end{gathered}
$$

Here, $\Delta$ and $\Delta^{\prime}$ are complete differentials. The geometric meanings of the Pfaff forms $\Delta$ and $\Delta^{\prime}$ are changing in the angles $\Phi$ and $\Phi^{\prime}$, respectively.

These results will be needed in next subsections.

Remark 14. Using two different moving planes $\mathscr{A}$ and $\mathscr{A}^{\prime}$ is an extension of the theory of moving coordinate system. We explain two-parameter planar Lorentzian motion $L / L^{\prime}$ by moving the coordinate system $\left\{A^{\prime} ; \mathbf{b}_{1}^{\prime}, \mathbf{b}_{2}^{\prime}\right\}$ to the coordinate system $\left\{A ; \mathbf{b}_{1}, \mathbf{b}_{2}\right\}$. Therefore, we can have the relation between the planes $\mathscr{A}$ and $\mathscr{A}^{\prime}$. In Section 3.8, we define the function that moves the point $A$ to the point $A^{\prime}$.

3.3. The Polar Axes of the Two-Parameter Planar Lorentzian Motion. In this section, we will analyze the polar axes of the motion $H_{I I}$ in two ways.

First way is by using the hyperbolic statements of the motion.

Theorem 15. The pole points of the motions $\mathrm{SH}_{I}$ obtained from $\mathrm{SH}_{I I}$ are on a time-like (space-like) line at each position in both moving and fixed planes. These lines are called the polar axes of the motion. 

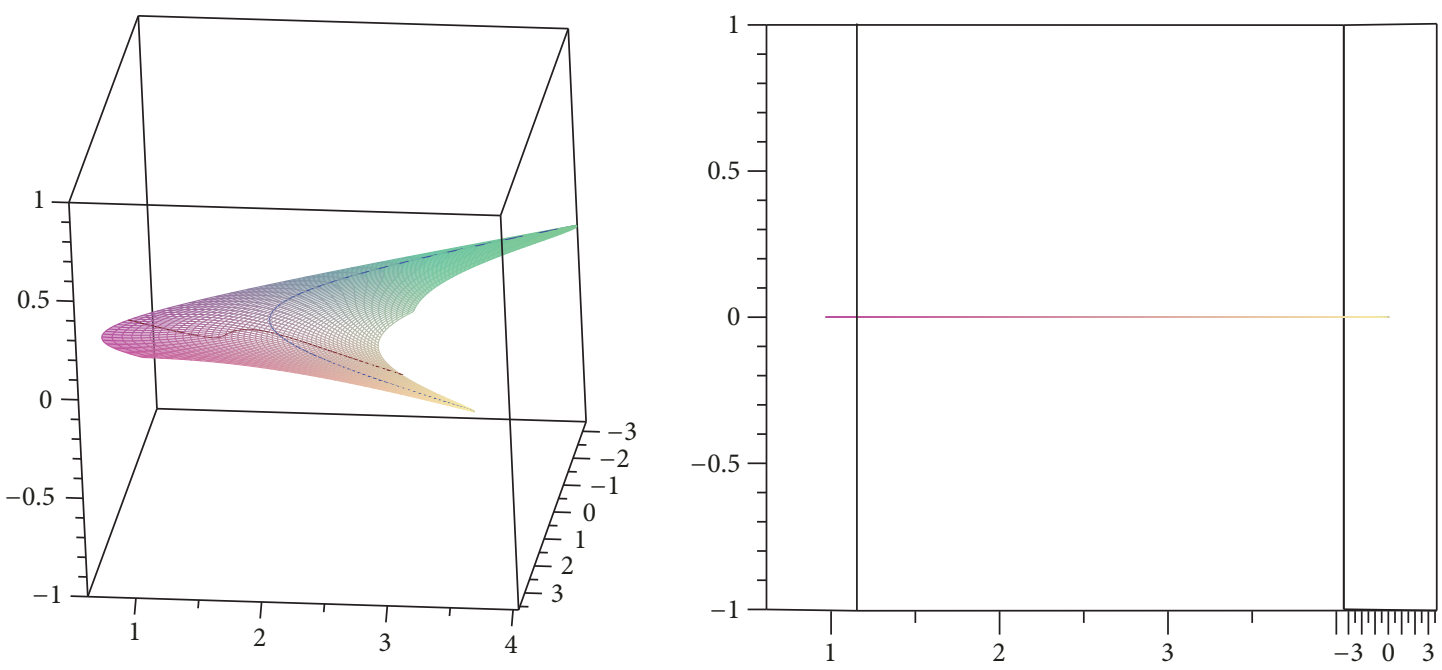

FIgURE 1: The example of the visualisation of the $H_{I I}$ and the motions $H_{I}$ obtained from $H_{I I}$.

Proof. Now, we will investigate the pole points $P$ of the motions $S H_{I}$ obtained from the motion $S H_{I I}$. Since $\mathbf{u}=$ $\mathbf{u}(\Theta, t)$ and $t=t(\Theta)$, we can write

$$
d \mathbf{u}=\frac{\partial \mathbf{u}}{\partial \Theta} d \Theta+\frac{\partial \mathbf{u}}{\partial t} \frac{d t}{d \Theta} d \Theta
$$

and

$$
\frac{d \mathbf{u}}{d \Theta}=\mathbf{u}_{\Theta}+\mathbf{u}_{t} \frac{d t}{d \Theta}
$$

Substituting last equality into (22), we have

$$
\begin{gathered}
\mathbf{p}=\mathbf{u}+j \mathbf{u}_{\Theta}+j \mathbf{u}_{t} \frac{d t}{d \Theta}, \\
\mathbf{p}^{\prime}=j e^{j \Theta} \mathbf{u}_{\Theta}+j e^{j \Theta} \mathbf{u}_{t} \frac{d t}{d \Theta} .
\end{gathered}
$$

If the position $(\Theta, t)$ of the motion $H_{I I}$ is fixed and $d t / d \Theta$ of the motion $H_{I}$ is changed, then $\mathbf{u}_{\Theta}$ is not equal to zero. We will denote by $g$ and $g^{\prime}$ the polar axes in the planes $L$ and $L^{\prime}$. From (33), it can be concluded that the pole axis $g$ passes through the point $\mathbf{p}_{0}=\mathbf{u}+j \mathbf{u}_{\Theta}$ and has the directrix vector $\mathbf{v}=j \mathbf{u}_{t}$. Similarly, the polar axis $g^{\prime}$ passes through the point $\mathbf{p}_{\mathbf{0}}^{\prime}=j e^{j \Theta} \mathbf{u}_{\Theta}$ and has the directrix vector $\mathbf{v}^{\prime}=j e^{j \Theta} \mathbf{u}_{t}=\mathbf{v} e^{j \Theta}$. Since, $\left\langle\mathbf{v}^{\prime}, \mathbf{v}^{\prime}\right\rangle_{L}=\langle\mathbf{v}, \mathbf{v}\rangle_{L}=-\left\langle\mathbf{u}_{t}, \mathbf{u}_{t}\right\rangle_{L}$, if $\mathbf{u}_{t}$ is a space-like (or a time-like) vector, then the polar axes $g$ and $g^{\prime}$ are time-like (or space-like) lines. These complete the proof.

Definition 16. The pole axes $g$ and $g^{\prime}$ correspond to each other in point-to-point. This is called the polar axis transformation.

Because of $\|\mathbf{v}\|_{L}=\left\|\mathbf{v}^{\prime}\right\|_{L}=\left|\mathbf{u}_{t}\right|$, the polar axes $g$ and $g^{\prime}$ are correspond to each other by equal velocity.

Second way is by using the moving coordinate system.

Theorem 17. The pole points of the motions $H_{I}$ obtained from $H_{I I}$ are on a time-like (space-like) line at each position in both moving and fixed planes. These lines are called the polar axes of the motion.

Proof. We can write

$$
\begin{aligned}
& p_{1}=\frac{\Sigma_{2}{ }^{\prime}-\Sigma_{2}}{\Delta^{\prime}-\Delta}, \\
& p_{2}=\frac{\Sigma_{1}{ }^{\prime}-\Sigma_{1}}{\Delta^{\prime}-\Delta},
\end{aligned}
$$

for the pole point $P=\left(p_{1}, p_{2}\right)$ of the motion $H_{I}$ obtained from the motion $H_{I I}$ with the help of the moving coordinate system. Since the Pfaff forms are functions of two-parameter $t_{1}$ and $t_{2}$, we can write

$$
\begin{aligned}
P\left(p_{1}, p_{2}\right)= & \left(K\left(t_{1}, t_{2}\right), M\left(t_{1}, t_{2}\right)\right) \\
& +\frac{1}{\delta}\left(L\left(t_{1}, t_{2}\right), N\left(t_{1}, t_{2}\right)\right),
\end{aligned}
$$

where $d t_{2} / d t_{1}=\delta$. Here, $K, M, L$, and $N$ are functions of the parameters $t_{1}$ and $t_{2}$. From (34), it can be said that the pole point $P$ is on a straight line passing through point $(K, M)$ and has the directrix vector $(L, N)$. If $(L, N)$ is a space-like (or a time-like) vector, then the polar axes $g$ and $g^{\prime}$ are space-like (or time-like) lines.

\subsection{The Density Invariance of The Polar Axis Transformation}

Theorem 18. The density of the time-like (or space-like) polar axis $g$ in the plane $L$ is equal to the density of the time-like (or space-like) polar axis $g^{\prime}$ in the plane $L^{\prime}$ under the motion $H_{I I}$ at any position.

Proof. The proof will be divided into two parts. We prove this theorem by using time-like and space-like polar axis separately.

(i) The Time-Like Polar Axis $g_{t}$. If we take that the polar axis coincides with the vector axis $\left\{A ; \mathbf{b}_{2}\right\}$, in Figure 2 , then we 


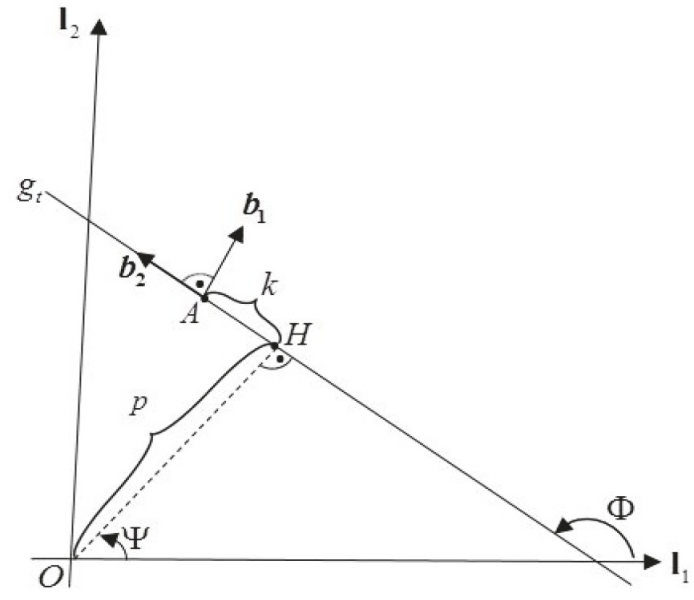

FIGURE 2: The polar axis $g_{t}$ coincides with the vector axis $\left\{A ; \mathbf{b}_{2}\right\}$.

have $p_{1}=0$ for the pole point $P=p_{1} \mathbf{b}_{1}+p_{2} \mathbf{b}_{2}$. There is no loss of generality in assuming it. Hence, $\Sigma_{2}^{\prime}-\Sigma_{2}=0$ is obtained from (34). If we take differentiation of the equality $\Sigma_{2}^{\prime}=\Sigma_{2}$ and use the integrability conditions, we find that

$$
\Sigma_{1}^{\prime} \wedge \Delta^{\prime}=\Sigma_{1} \wedge \Delta
$$

Since we can write

$$
\mathbf{a}=\mathbf{A O}=-p \mathbf{b}_{1}-k \mathbf{b}_{2},
$$

we get the differentiation of the point in the plane $\mathscr{A}$ as

$$
\begin{aligned}
d \mathbf{a} & =-d p \mathbf{b}_{1}-d k \mathbf{b}_{2}-p d \mathbf{b}_{1}-k d \mathbf{b}_{2} \\
& =-(d p+k \Delta) \mathbf{b}_{1}-(d k+p \Delta) \mathbf{b}_{2} .
\end{aligned}
$$

Considering the derivative equations, we can write

$$
\begin{aligned}
& \Sigma_{1}=-(d p+k \Delta) \\
& \Sigma_{2}=-(d k+p \Delta) .
\end{aligned}
$$

Then, using $\Delta=d \Phi=d \Psi$ and $d p=k \Delta-\Sigma_{1}$, we obtain the following equation for the density of the polar axis $g_{t}$ :

$$
d g_{t}=d p \wedge \Psi=-\Sigma_{1} \wedge \Delta .
$$

The same proof works for the density of the polar axis $g_{t}^{\prime}$

$$
d g_{t}^{\prime}=d p^{\prime} \wedge \Psi^{\prime}=-\Sigma_{1}{ }^{\prime} \wedge \Delta^{\prime}
$$

in the plane $L^{\prime}$. This finishes the proof.

(ii) The Space-Like Polar Axis $g_{s}$. In analogy to the proof of the above part, it can be shown that $d g_{s}=d g_{s}^{\prime}$.

\subsection{The Two-Parameter Planar Lorentzian Motion with Zero- the Density of the Polar Axis}

Theorem 19. The two-parameter planar Lorentzian motion $H_{I I}$ with zero-the density of time-like (space-like) pole axis is defined by contacting the evolute space-like (time-like) curve $W$ to its envelope space-like (time-like) curve $W^{\prime}$.

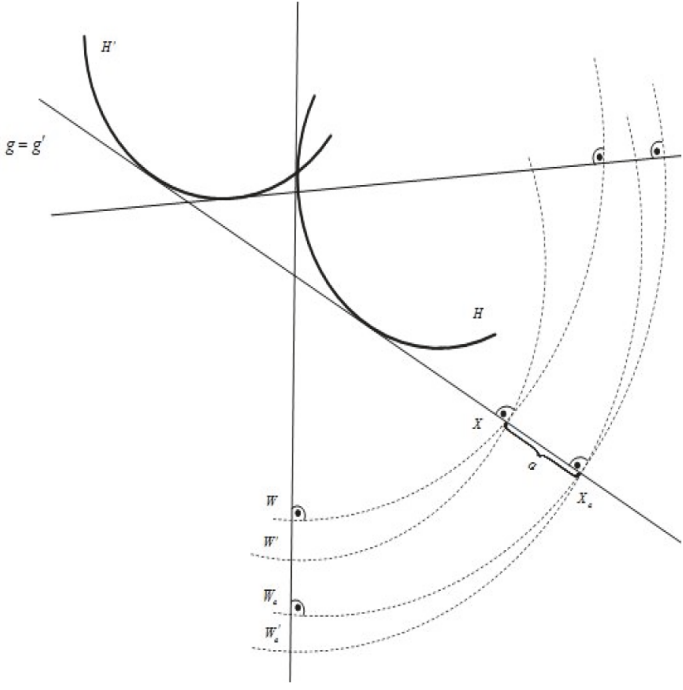

FIgURE 3: The envelope and evolute curves of the polar axes.

Proof. We assume that the non null polar axes of the motion $H_{I I}$ have zero-density. In this case,

$$
d g=d p \wedge d \Psi=0
$$

and

$$
d g^{\prime}=d p^{\prime} \wedge d \Psi^{\prime}=0
$$

can be written. So that, $d p, d \Psi$ and $d p^{\prime}, d \Psi^{\prime}$ are linearly dependent. Thus, $p$ can be expressed as a function of $\Psi, p=$ $p(\Psi)$. Likewise, we can write that $p^{\prime}=p^{\prime}\left(\Psi^{\prime}\right)$. The functions $p$ and $p^{\prime}$ define the one-parameter families of the non null lines $g, g^{\prime}$ in the Lorentzian planes $L, L^{\prime}$, respectively. Let $H$ and $H^{\prime}$ be the envelope curves of the lines $p(\Psi), p^{\prime}(\Psi)^{\prime}$, respectively. Let $W$ be an orthogonal curve to the family of lines $g$. Since the tangent of $W$ is perpendicular to the tangent of $H$, and the curve $W$ is called the evolute of the envelope $H$, Figure 3. As a consequence of these, we can say "the motion $H_{I I}$ with zero pole axis density is defined by contacting the evolute curve $W$ to its envelope curve $W^{\prime}$." If the pole axis $g$ is a time-like (or a space-like) line, then the envelope $H$ will be a time-like (or a space-like) and hence the evolute curve $W$ will be a space-like (or a time-like).

If we take the curves $W_{a}$ and $W_{a}^{\prime}$ which have distance $a$ from the curves $W$ and $W^{\prime}$, the same $H_{I I}$ motion is obtained. The motion $H_{I I}$ is independent from the choice of the distance $a$.

Moreover, the motion can also be explained as follows.

The curves $H \in L$ and $H^{\prime} \in L^{\prime}$ roll without sliding on the pole axis $g=g^{\prime}$. Because of the decomposability of the motion $H_{I I}$ into two independent rolling movements, the motion is also called the separable.

\subsection{The Curve Plotter Points}

Theorem 20. The pole axes of the two-parameter planar Lorentzian motion are also the locus of the curve plotter points of the motion $H_{I I}$. 
Proof. Since the sliding velocity of the point $X$ is

$$
\begin{aligned}
d_{f} \mathbf{x}= & \left\{\left(\Sigma_{1}-\Sigma_{1}{ }^{\prime}\right)+y\left(\Delta^{\prime}-\Delta\right)\right\} \mathbf{b}_{1} \\
& +\left\{\left(\Sigma_{2}-\Sigma_{2}{ }^{\prime}\right)+x\left(\Delta^{\prime}-\Delta\right)\right\} \mathbf{b}_{2},
\end{aligned}
$$

we can write the following equation for the density of the point $X$ under the motion $H_{I I}$ using the moving coordinate system $\left\{A ; \mathbf{b}_{1}, \mathbf{b}_{2}\right\}$ :

$$
\begin{aligned}
d X^{\prime}= & x\left(\Sigma_{1}-\Sigma_{1}^{\prime}\right) \wedge\left(\Delta^{\prime}-\Delta\right)+y\left(\Delta^{\prime}-\Delta\right) \\
& \wedge\left(\Sigma_{2}-\Sigma_{2}^{\prime}\right)+\left(\Sigma_{1}-\Sigma_{1}^{\prime}\right) \wedge\left(\Sigma_{2}-\Sigma_{2}^{\prime}\right) .
\end{aligned}
$$

If the Pfaff forms $\Sigma_{1}-\Sigma_{1}^{\prime}, \Sigma_{2}-\Sigma_{2}^{\prime}$, and $\Delta^{\prime}-\Delta$ are linearly dependent, or $\Delta^{\prime}-\Delta=0$, the density of the point $X$ is zero. In these cases, we can see that these points are on the pole axis. These points behave as if they were in one-parameter motion and they describe a curved element, not a surface element. Hence, they are named as the curve plotter points of the motion $H_{I I}$. Using (34), we can also say that the density of the pole points $P=\left(p_{1}, p_{2}\right)$ is zero.

As a summary during the motion $H_{I I}$, only the pole points on the polar axis move with one-parameter.

\subsection{The Determination of the Two-Parameter Planar Lorentzi- an Motion Using the Polar Axes}

Theorem 21. The non null line transformation $g \longrightarrow g^{\prime}(g \in$ $\left.L, g^{\prime} \in L^{\prime}\right)$, which preserves the density of the lines, is a member of a family of two-parameter planar Lorentzian motions.

Proof. The proof falls naturally into two parts. We will prove this theorem by using the time-like and space-like polar axis separately.

(i) The Time-Like Polar Axis $g_{t}$. Without loss of generality, we can introduce the moving coordinate systems $\left\{A ; \mathbf{b}_{1}, \mathbf{b}_{2}\right\}$, $\left\{A^{\prime} ; \mathbf{b}_{1}{ }^{\prime}, \mathbf{b}_{2}{ }^{\prime}\right\}$ in the Lorentzian planes $L$ and $L^{\prime}$ such that the time-like polar axis $g_{t}$ coincides with the vector axis $\left\{A ; \mathbf{b}_{2}\right\}$ in $L$ and the time-like polar axis $g_{t}^{\prime}$ coincides with the vector axis $\left\{A^{\prime} ; \mathbf{b}_{2}^{\prime}\right\}$ in $L^{\prime}$. Since the transformation preserves the density of lines, we can write that

$$
d \Sigma_{2}=d \Sigma_{2}^{\prime}
$$

We will make the following assumption: the origin of the coordinate system $\left\{A^{\prime} ; \mathbf{b}_{1}{ }^{\prime}, \mathbf{b}_{2}{ }^{\prime}\right\}$ moves to the point $C^{\prime}$ along the pole axis $g^{\prime}$ in the plane $L^{\prime}$. In this case, we have the coordinate system $\left\{C^{\prime} ; \mathbf{b}_{1}{ }^{\prime}, \mathbf{b}_{2}{ }^{\prime}\right\}$ at this point, Figure 4 . Now, we can explain two-parameter planar Lorentzian motion by moving the coordinate system $\left\{C^{\prime} ; \mathbf{b}_{1}^{\prime}, \mathbf{b}_{2}^{\prime}\right\}$ to the coordinate system $\left\{A=C ; \mathbf{b}_{1}, \mathbf{b}_{2}\right\}$.

We have the vector equations

$$
\mathbf{c}^{\prime}=\mathbf{C}^{\prime} \mathbf{O}^{\prime}=\mathbf{C}^{\prime} \mathbf{A}^{\prime}+\mathbf{A}^{\prime} \mathbf{O}^{\prime}
$$

or

$$
\mathbf{c}^{\prime}=\mathbf{a}^{\prime}+q \mathbf{b}_{2}{ }^{\prime} \text {. }
$$

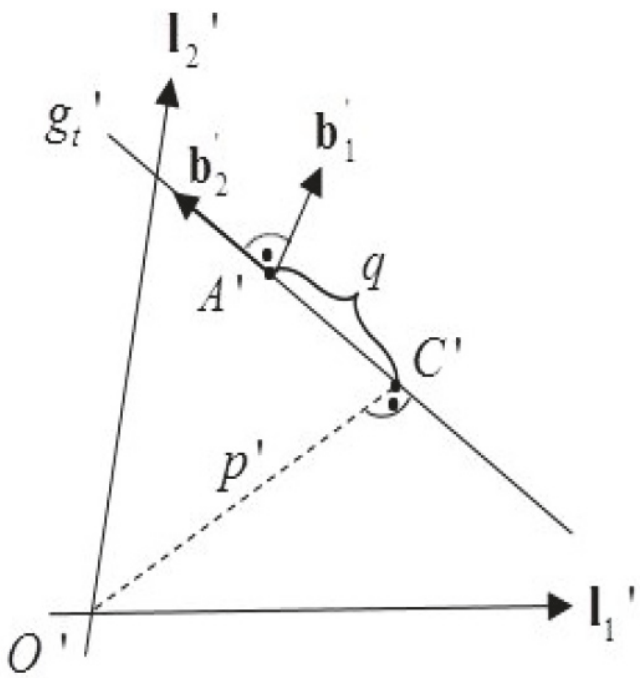

FIGURE 4: The polar axis $g_{t}^{\prime}$ coincides with the vector axis $\left\{A^{\prime} ; \mathbf{b}_{2}^{\prime}\right\}$.

Then, the differentiation of the point $C^{\prime}$ can be written as follows:

$$
d \mathbf{c}^{\prime}=w_{1}^{\prime} \mathbf{b}_{1}^{\prime}+w_{2}^{\prime} \mathbf{b}_{2}^{\prime}=\left(\Sigma_{1}^{\prime}+q \Delta^{\prime}\right) \mathbf{b}_{1}^{\prime}+\left(\Sigma_{2}^{\prime}+d q\right) \mathbf{b}_{2}^{\prime} .
$$

By writing $d q=w_{2}^{\prime}-\Sigma_{2}^{\prime}$, we can assert that $w_{2}^{\prime}=\Sigma_{2}$, because the polar axis $g_{t}$ coincides with the vector axis $\left\{A ; \mathbf{b}_{2}\right\}$ and the polar axis $g_{t}^{\prime}$ coincides with the vector axis $\left\{C^{\prime} ; \mathbf{b}_{2}^{\prime}\right\}$. In this case, we obtain that

$$
d q=\Sigma_{2}-\Sigma_{2}^{\prime}
$$

or

$$
d q=A\left(t_{1}, t_{2}\right) d t_{1}+B\left(t_{1}, t_{2}\right) d t_{2} .
$$

Since the Pfaff forms $\Sigma_{2}, \Sigma_{2}^{\prime}$ satisfy the integrability conditions, $q$ is a complete differential. The function $q$ can be obtained by integrating $d q$. Without loss of generality, let us assume that $t_{2}$ is constant. Under this assumption, if we integrate $d q$ over the parameter $t_{1}$, then the function $q$ is obtained as

$$
q=\int A\left(t_{1}, t_{2}\right) d t_{1}+c\left(t_{2}\right)
$$

In this case, the function $q$ is determined with the parameter $t_{2}$, which proves the theorem.

(ii) The Space-Like Polar Axis $g_{s}$. If we take that the space-like polar axis $g_{s}$ coincides with the vector axis $\left\{A ; \mathbf{b}_{1}\right\}$ and the space-like polar axis $g_{s}^{\prime}$ coincides with the vector axis $\left\{A^{\prime}=\right.$ $\left.C^{\prime} ; \mathbf{b}_{1}{ }^{\prime}\right\}$, then we obtain that

$$
d q=\Sigma_{1}-\Sigma_{1}^{\prime}
$$

where the Pfaff is the differential form of the parameters $t_{1}$ and $t_{2}$. The same proof works for this case. 


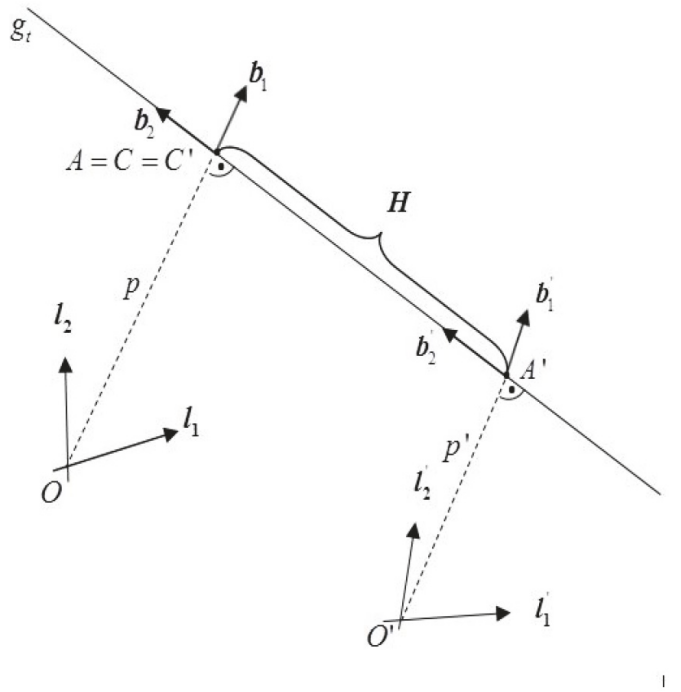

FIGURE 5: The support function on the polar axis $g_{t}$.

3.8. The Support Function. We have divided the support function into two parts. We give it by using time-like and space-like polar axis, separately.

(i) The Time-Like Polar Axis $g_{t}$. We take the moving coordinate systems $\left\{A=C=C^{\prime} ; \mathbf{b}_{1}, \mathbf{b}_{2}\right\},\left\{A^{\prime} ; \mathbf{b}_{1}{ }^{\prime}, \mathbf{b}_{2}{ }^{\prime}\right\}$ in the Lorentzian planes $L$ and $L^{\prime}$ such that the time-like polar axis $g_{t}$ coincides with the vector axis $\left\{A=C=C^{\prime} ; \mathbf{b}_{2}\right\}$ and the time-like polar axis $g_{t}^{\prime}$ coincides with the vector axis $\left\{A^{\prime} ; \mathbf{b}_{2}^{\prime}\right\}$. There is no loss of generality in assuming that the origin of the $\left\{A^{\prime} ; \mathbf{b}_{1}{ }^{\prime}, \mathbf{b}_{2}{ }^{\prime}\right\}$ moves to the point $A=C=C^{\prime}$, Figure 5 .

We can write the vector equation

$$
\mathbf{a}=\mathbf{A O}=-p \mathbf{b}_{1}
$$

By differentiating this equation, we see that

$$
\begin{aligned}
& \Sigma_{1}=-d p, \\
& \Sigma_{2}=-p \Delta .
\end{aligned}
$$

Similarly,

$$
\begin{aligned}
& \Sigma_{1}^{\prime}=-d p^{\prime}, \\
& \Sigma_{2}^{\prime}=-p^{\prime} \Delta^{\prime}
\end{aligned}
$$

can be obtained in the plane $L^{\prime}$, so that a special case of the function q, defined in Section 3.6, can be written as follows:

$$
\mathbf{H}=\int\left(-p \Delta+p^{\prime} \Delta^{\prime}\right)=\int\left(-p d \Phi+p^{\prime} d \Phi^{\prime}\right) .
$$

(ii) The Space-Like Polar Axis $g_{s}$. If we take that the space-like polar axis $g_{s}$ coincides with the vector axis $\left\{A=C=C^{\prime} ; \mathbf{b}_{1}\right\}$ and the space-like polar axis $g_{s}^{\prime}$ coincides with the vector axis $\left\{A^{\prime} ; \mathbf{b}_{1}{ }^{\prime}\right\}$, then we obtain that

$$
\mathbf{H}=\int\left(p \Delta-p^{\prime} \Delta^{\prime}\right)=\int\left(p d \Phi-p^{\prime} d \Phi^{\prime}\right) .
$$

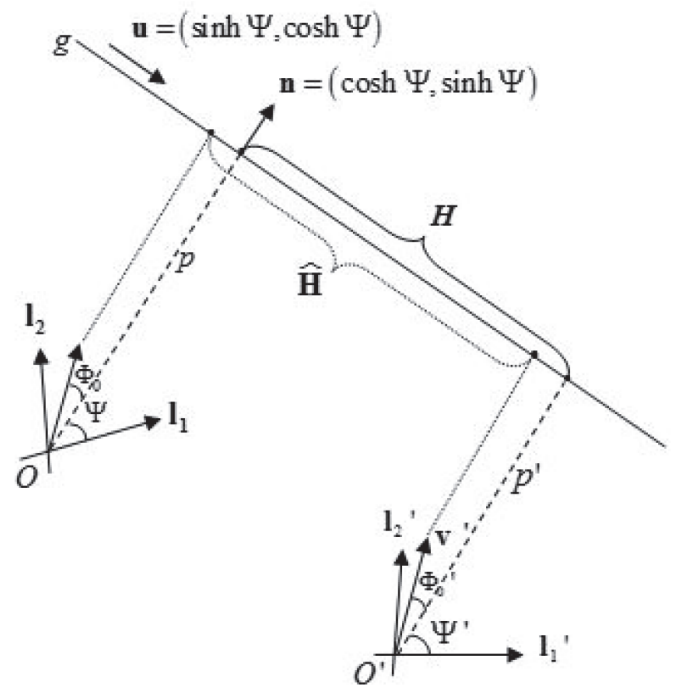

FIGURE 6: The change of the support function on the polar axis $g_{t}$.

Definition 22. Assuming $\Delta \wedge \Delta^{\prime} \neq 0$, we will consider the angles $\Phi$ and $\Phi^{\prime}$ as parameters instead of $t_{1}$ and $t_{2}$. Thus, if we derive (57) and (58) with respect to the parameters $\Phi$ and $\Phi^{\prime}$, we get

$$
\begin{aligned}
\mathbf{H}_{\Phi} & =-p, \\
\mathbf{H}_{\Phi^{\prime}} & =p^{\prime} \\
\mathbf{H}_{\Phi} & =p, \\
\mathbf{H}_{\Phi^{\prime}} & =-p^{\prime}
\end{aligned}
$$

for the time-like and space-like pole axes, respectively. The function $\mathbf{H}\left(\Phi, \Phi^{\prime}\right)$ is called the support function of the twoparameter planar Lorentzian motion.

Let us examine how the support function $\mathbf{H}$ changes when the vectors $\mathbf{v}=v_{1} \mathbf{l}_{1}+v_{2} \mathbf{l}_{2} \in L$ and $\mathbf{v}^{\prime}=v_{1}^{\prime} \mathbf{l}_{1}{ }^{\prime}+v_{2}^{\prime} \mathbf{l}_{2}{ }^{\prime} \in L^{\prime}$ rotate by the angles $\Phi_{0}$ and $\Phi_{0}^{\prime}$, respectively. We investigate this by time-like and space-like polar axis separately.

(i) The Time-Like Polar Axis $g_{t}$. If we take into consideration the time-like polar axis $g_{t}$, Figure 6 , the following change is obtained:

$$
\begin{aligned}
\hat{\mathbf{H}}= & \mathbf{H}+\left(v_{1} \sinh \Psi-v_{2} \cosh \Psi\right) \\
& -\left(v_{1}^{\prime} \sinh \Psi^{\prime}-v_{2}^{\prime} \cosh \Psi^{\prime}\right) .
\end{aligned}
$$

Moreover, the differentiation of the function $\hat{\mathbf{H}}$ can be written as follows:

$$
\begin{aligned}
d \hat{\mathbf{H}}= & d \mathbf{H}+v_{1} \cosh \Psi d \Psi-v_{2} \sinh \Psi d \Psi \\
& -v_{1}^{\prime} \cosh \Psi^{\prime} d \Psi^{\prime}+v_{2}^{\prime} \sinh \Psi^{\prime} d \Psi^{\prime}
\end{aligned}
$$


If $\hat{p}=p-v_{1} \cosh \Psi+v_{2} \sinh \Psi$ and $\hat{p^{\prime}}=p^{\prime}-v_{1}^{\prime} \cosh \Psi^{\prime}+$ $v_{2}^{\prime} \sinh \Psi^{\prime}$ are taken,

$$
d \hat{\mathbf{H}}=-\hat{p} d \Phi+\hat{p}^{\prime} d \Phi^{\prime}
$$

is obtained and

$$
\hat{\mathbf{H}}=\int\left(-\hat{p} d \Phi+\hat{p^{\prime}} d \Phi^{\prime}\right)
$$

can be written, where $\hat{p}$ is the distance of $\mathbf{v}$ to the time-like polar axis $g_{t}$ and $\hat{p^{\prime}}$ is the distance of $\mathbf{v}^{\prime}$ to the time-like polar axis $g_{t}^{\prime}$.

(ii) The Space-Like Polar Axis $g_{s}$. If we take into consideration the space-like polar axis $g_{s}$, then the following statement is obtained:

$$
\hat{\mathbf{H}}=\int\left(\hat{p} d \Phi-\hat{p}^{\prime} d \Phi^{\prime}\right),
$$

where $\hat{p}$ is the distance of $\mathbf{v}$ to the space-like polar axis $g_{s}$ and $\hat{p^{\prime}}$ is the distance of $\mathbf{v}^{\prime}$ to the space-like polar axis $g_{s}^{\prime}$.

\section{Conclusion}

After defining the hyperbolic statement of the two-parameter planar Lorentzian motion $H_{I I}$, the geometric aspects of the motion $H_{I I}$ are investigated by considering the polar axes and the moving coordinate system. Thereafter using the polar axes the two-parameter planar Lorentzian motion is ascertained. Taking advantage of those verities, our next study will be concentrated on the main one-parameter motions, the geodesic motions, the osculator motions and the sliding motions obtained from $H_{I I}$. Eventually, we have faith in that study whose viewpoint sheds some new lights on the study of motions in Euclidean and non-Euclidean planes.

\section{Data Availability}

No data were used to support this study.

\section{Conflicts of Interest}

The authors declare that they have no conflicts of interest.

\section{Acknowledgments}

This work was supported by Research Fund of the Yildiz Technical University, Project no. FDK-2018-3320. G. Y. Şentürk has been partially supported by TÜBİTAK (2211Domestic Ph.D. Scholarship), The Scientific and Technological Research Council of Turkey.

\section{References}

[1] W. Blaschke and H. R. Müller, Ebene Kinematik, Verlag Von R. Oldenbourg, München, 1956.
[2] A. A. Ergin, "On the one-parameter Lorentzian Motion," Commun. Fac. Sci. Univ. Ank., Serias A, vol. 40, pp. 59-66, 1991.

[3] M. Akar, S. Yüce, and N. Kuruoğlu, "One-parameter planar motion on the Galilean plane," International Electronic Journal of Geometry, vol. 6, no. 1, pp. 79-88, 2013.

[4] A. A. Ergin, "Three Lorentzian planes moving with respect to one another and pole points," Commun. Fac. Sci.Univ.Ank. Series A, vol. 41, pp. 79-84, 1992.

[5] N. Bayrak Gürses and S. Yüce, "One-parameter planar motions in affine Cayley-Klein planes," European Journal of Pure and Applied Mathematics, vol. 7, no. 3, pp. 335-342, 2014.

[6] S. Şahin and S. Yüce, "Higher-order accelerations and poles under the one-parameter planar hyperbolic motions and their inverse motions," Mathematical Problems in Engineering, Art. ID 686509, 8 pages, 2014.

[7] M. M. Stanisic and G. R. Pennock, "The canonical invers velocity and acceleration solutions of a planar two link open chain," International Journal of Robotics Research, vol. 5, no. 2, pp. 82-90, 1986.

[8] O. Bottema and B. Roth, Theoretical kinematics, vol. 24 of NorthHolland Series in Applied Mathematics and Mechanics, NorthHolland Publishing Co., Amsterdam-New York, 1979.

[9] C. G. Gibson, W. Hawes, and C. A. Hobbs, "Local pictures for general two-parameter planar motions," in Advances in robot kinematics and computational geometry (Ljubljana, 1994), pp. 49-58, Kluwer Acad. Publ., Dordrecht, 1994.

[10] C. G. Gibson, D. Marsh, and Y. Xiang, "Singular aspects of general planar motions with two degrees of freedom," International Journal of Robotics Research, vol. 17, no. 10, pp. 1068-1080, 1998.

[11] M. K. Karacan and Y. Yayl, "General two parameter motion," Algebras, Groups and Geometries, vol. 22, no. 1, pp. 137-144, 2005.

[12] M. K. Karacan, Kinematic applications of two parameter motions [Ph.D. Thesis], Ankara University, Graduate School, 2004.

[13] M. Çelik and M. A. Güngör, "Two parameter motions on the Galilean plane," in Proceedings of the 3rd International Eurasian Conference on Mathematical Sciences and Applications, 2014.

[14] M. K. Karacan and Y. Yayl,, "Special two parameter motion in Lorentzian plane," Thai Journal of Mathematics, vol. 2, no. 2, pp. 239-246, 2004.

[15] M. K. Karacan and Y. Yaylı, "Special two parameter motion," Mathematical and Computational Applications, vol. 10, no. 1, pp. 27-34, 2005.

[16] D. Ünal, M. Çelik, and M. A. Güngör, "On the two parameter motions in the complex plane," University Politehnica of Bucharest Scientific Bulletin Series A Applied Mathematics and Physics, vol. 2, pp. 185-194, 2013.

[17] M. Çelik and M. A. Güngör, "Two parameter Hyperbolic motions," in Proceedings of the Eurasian Conference on Mathematical Sciences and Applications, Sarajevo, Bosnia And Herzegovina, 2013.

[18] L. Tsai, "Instantaneous kinematics of a special two-parameter motion," Journal of Engineering for Industry, vol. 99, no. 2, pp. 336-340, 1977.

[19] M. Hlavova, "Two-parametric motions in the Lobatchevski plane," Journal for Geometry and Graphics, vol. 6, no. 1, pp. 2735, 2002.

[20] J. J. Shi, M. Alenezy, I. V. Smirnova, and M. Bilgen, "Construction of a two-parameter empirical model of left ventricle wall motion using cardiac tagged magnetic resonance imaging data," Biomedical Engineering OnLine, 11:79, 2012. 
[21] Q. Grimal, G. Rus, W. J. Parnell, and P. Laugier, "A twoparameter model of the effective elastic tensor for cortical bone," Journal of Biomechanics, vol. 44, no. 8, pp. 1621-1625, 2011.

[22] G. S. Birman and K. Nomizu, "Trigonometry in Lorentzian geometry," The American Mathematical Monthly, vol. 91, no. 9, pp. 543-549, 1984.

[23] G. S. Birman, "Crofton's and Poincaré's formulas in the Lorentzian plane," Geometriae Dedicata, vol. 15, no. 4, pp. 399411, 1984.

[24] L. Santaló, Integral Geometry and Geometric Probability, Cambridge University Press, 2nd edition, 2004.

[25] S. Yüce and N. Kuruoğlu, "Cauchy formulas for enveloping curves in the Lorentzian plane and Lorentzian kinematics," Results in Mathematics, vol. 54, no. 1-2, pp. 199-206, 2009.

[26] S. Yüce and N. Kuruoğlu, "One-parameter plane hyperbolic motions," Advances in Applied Clifford Algebras (AACA), vol. 18, no. 2, pp. 279-285, 2008.

[27] G. Sobczyk, "The Hyperbolic Number Plane," The College Mathematics Journal, vol. 26, no. 4, pp. 268-280, 1995. 


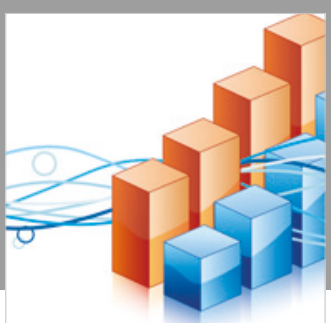

Advances in

Operations Research

\section{-n-m}
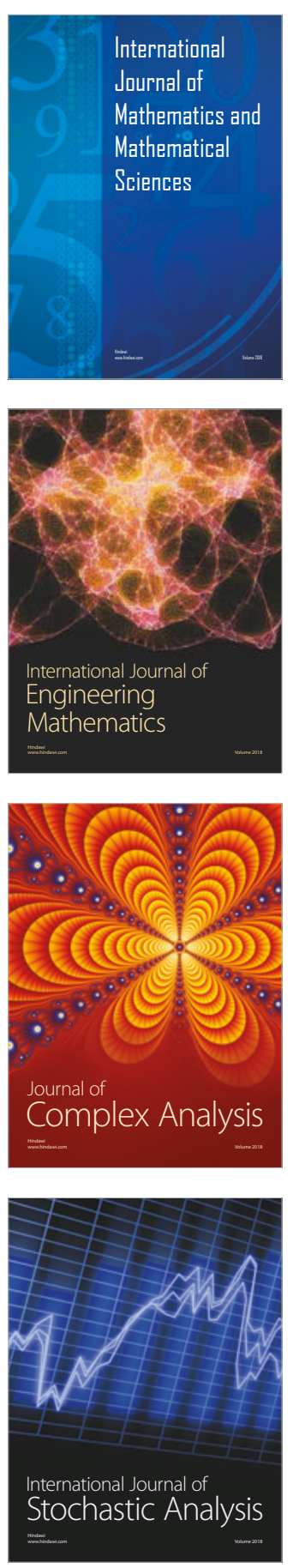
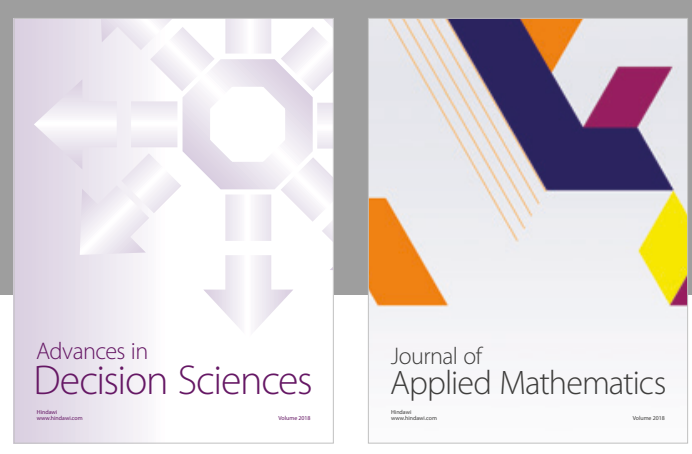

Journal of

Applied Mathematics
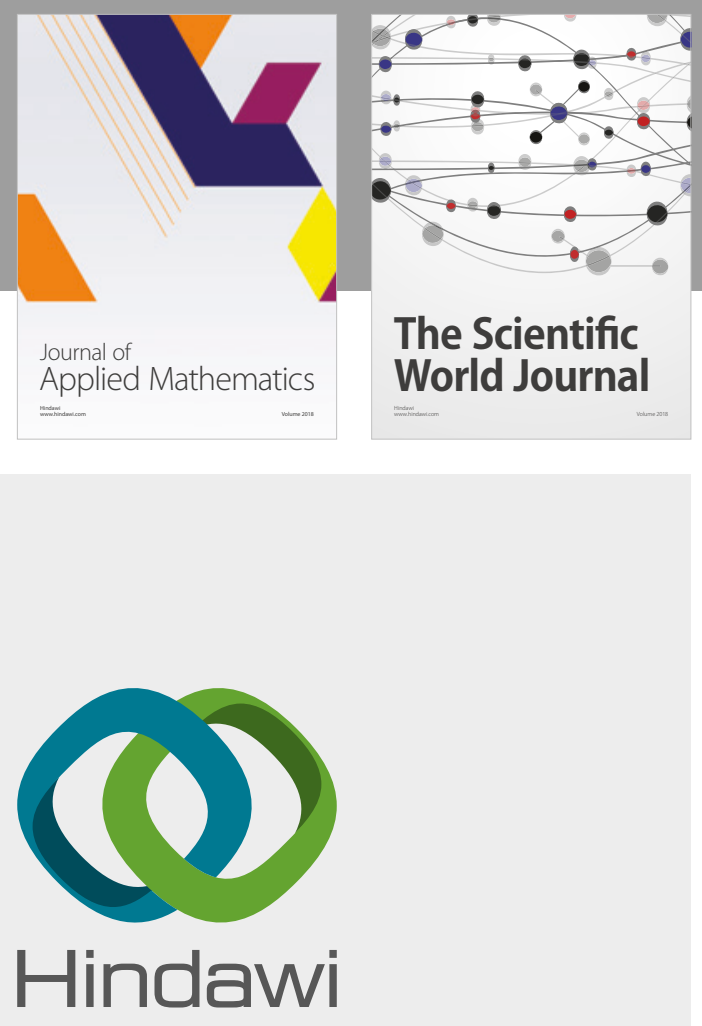

Submit your manuscripts at

www.hindawi.com

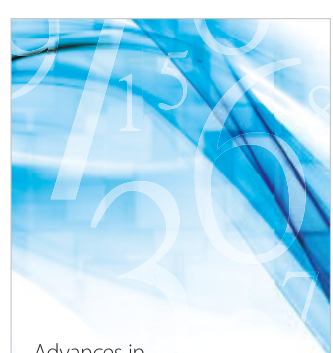

Advances in
Numerical Analysis
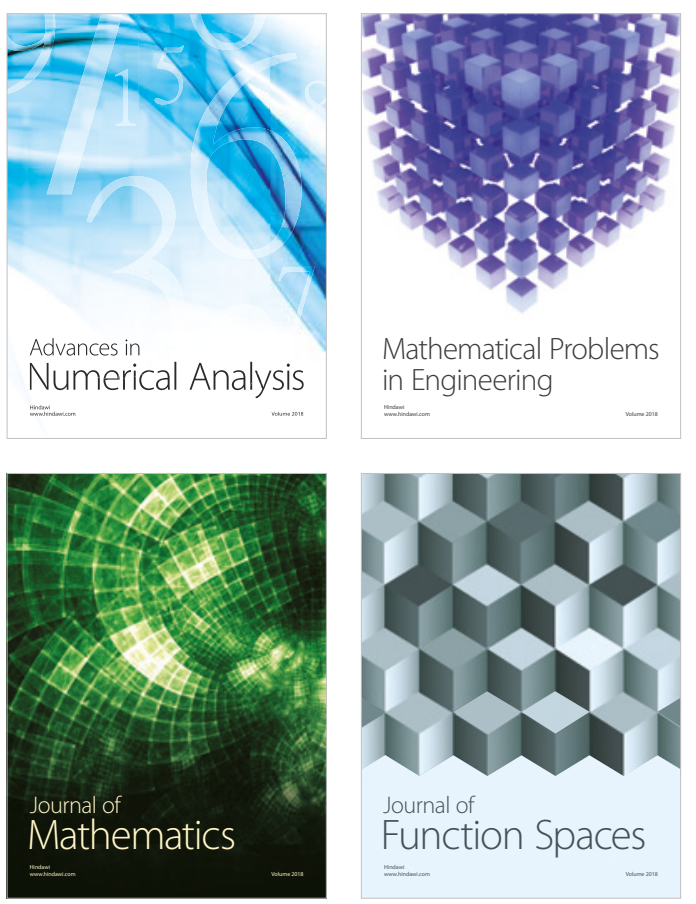

Mathematical Problems in Engineering

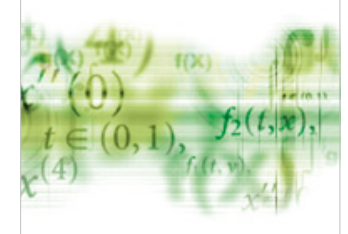

International Journal of

Differential Equations

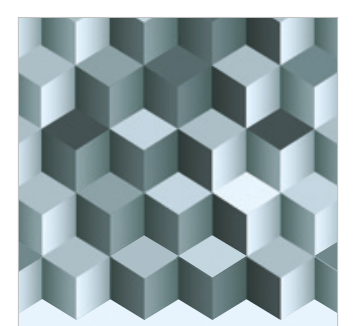

Journal of

Function Spaces

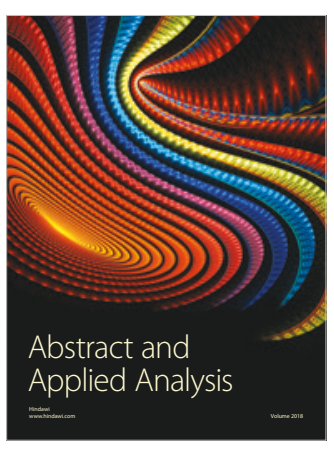

The Scientific

World Journal

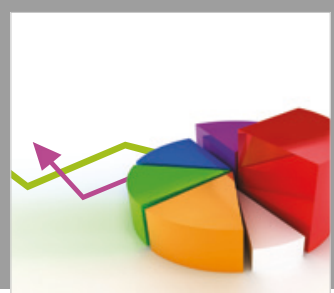

Journal of

Probability and Statistics
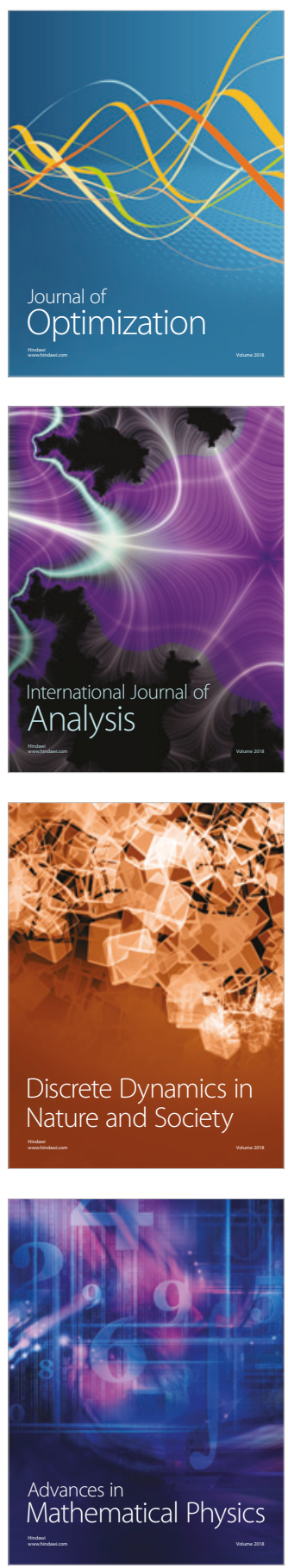